\title{
DOUBLE STAR RECOGNITION
}

\author{
M.FRGESCHLE, F. MIGNARD AND J.L. FALIN \\ OCA / CERGA \\ Avenue Copernic \\ 06130 Grasse \\ France
}

\begin{abstract}
Hipparcos observations provide a wealthy source of discovery of new double and multiple stars. We have developed adequate procedures to recognize in the signal parameters the multiplicity of the stellar source passing through the Hipparcos field of view. These procedures are outlined together with the design of the statistical tests employed. Sampling on real data indicates that about 6000 new double stars will be detected at the mission completion.
\end{abstract}

